\title{
On the Existence of Tree Backbones that Realize the Chromatic Number on a Backbone Coloring
}

\author{
J. Araujo and A. A. Cezar and A. Silva \\ ParGO Group - Parallellism, Graphs and Optimization \\ Departamento de Matemática \\ Universidade Federal do Ceará, Fortaleza, Brazil
}

October 17, 2018

\begin{abstract}
A proper $k$-coloring of a graph $G=(V, E)$ is a function $c: V(G) \rightarrow$ $\{1, \ldots, k\}$ such that $c(u) \neq c(v)$, for every $u v \in E(G)$. The chromatic number $\chi(G)$ is the minimum $k$ such that there exists a proper $k$-coloring of $G$. Given a spanning subgraph $H$ of $G$, a $q$-backbone $k$-coloring of $(G, H)$ is a proper $k$-coloring $c$ of $V(G)$ such that $|c(u)-c(v)| \geq q$, for every edge $u v \in E(H)$. The $q$-backbone chromatic number $B B C_{q}(G, H)$ is the smallest $k$ for which there exists a $q$-backbone $k$-coloring of $(G, H)$. In this work, we show that every connected graph $G$ has a spanning tree $T$ such that $B B C_{q}(G, T)=\max \left\{\chi(G),\left\lceil\frac{\chi(G)}{2}\right\rceil+q\right\}$, and that this value is the best possible.

As a direct consequence, we get that every connected graph $G$ has a spanning tree $T$ for which $B B C_{2}(G, T)=\chi(G)$, if $\chi(G) \geq 4$, or $B B C_{2}(G, T)=\chi(G)+1$, otherwise. Thus, by applying the Four Color Theorem, we have that every connected nonbipartite planar graph $G$ has a spanning tree $T$ such that $B B C_{2}(G, T)=4$. This settles a question by Wang, Bu, Montassier and Raspaud (2012), and generalizes a number of previous partial results to their question.
\end{abstract}

\section{Introduction}

For basic notions and terminology on Graph Theory, the reader is referred to [1]. All graphs in this work are considered to be simple. Because we investigate the existence of a spanning tree with certain property, we also consider only connected graphs. However, for disconnected graphs, the statements hold by replacing "spanning tree" by "spanning forest". A proper $k$-coloring of a graph $G$ is a function $c: V(G) \rightarrow\{1, \ldots, k\}$ such that $c(u) \neq c(v)$, for every $u v \in$ $E(G)$. If $G$ admits a proper $k$-coloring, we say that $G$ is $k$-colorable. The chromatic number of $G$, denoted by $\chi(G)$, is the smallest positive integer $k$ such that $G$ is $k$-colorable. Determining the chromatic number of a graph is an 
NP-hard problem on Karp's list [10] and one of the most studied problems on Graph Theory [9, 11].

Given a spanning subgraph $H$ of $G$, and positive integers $k$ and $q$, a $q$ backbone $k$-coloring of $(G, H)$ is a proper $k$-coloring $c$ of $G$ such that $\mid c(u)$ $c(v) \mid \geq q$, for every $u v \in E(H)$. The q-backbone chromatic number of $(G, H)$, denoted by $B B C_{q}(G, H)$, is the smallest integer $k$ for which $(G, H)$ admits a $q$-backbone $k$-coloring.

This parameter was first introduced by Broersma et al. 2] as a model for the frequency assignment problem where certain channels of communication are more demanding than others. In their seminal work, they only considered $q=2$ and they were interested in finding out how far away from $\chi(G)$ can $B B C_{2}(G, H)$ be in the worst case. Concerning trees, for each positive integer $k$, they defined:

$$
\mathcal{T}_{k}=\max \left\{B B C_{2}(G, T): \chi(G)=k \text { and } T \text { is a spanning tree of } G\right\} .
$$

Note that, if $c$ is a proper $\chi(G)$-coloring of $G$, then by recoloring each vertex $u$ with color $2 c(u)-1$, we obtain a proper $(2 \chi(G)-1)$-coloring of $G$ where every color is odd. Therefore, we get $B B C_{2}(G, G) \leq 2 \chi(G)-1$. This gives an upper bound of $2 k-1$ for $\mathcal{T}_{k}$. In [2], they proved that this is actually best possible.

Theorem 1 (Broersma et al. [2]). $\mathcal{T}_{k}=2 k-1$, for every positive integer $k$.

This means that, between all the $k$-colorable graphs, there is one that attains this upper bound. However, it does not give any insight on how bad can a tree backbone be for a given graph $G$. One could then define $\mathcal{T}_{2}(G)$ as the maximum $B B C_{2}(G, T)$, where $T$ is a spanning tree of $G$. This worst case behaviour has been studied for planar graphs. If $G$ is planar, because $\chi(G) \leq 4$ and the fact that $B B C_{2}(G, G) \leq 2 \chi(G)-1$, we get $\mathcal{T}_{2}(G) \leq 7$. Broersma et al. 3 give examples where $B B C_{2}(G, T)=6$, and conjecture that $\mathcal{T}_{2}(G)=6$. A partial result for their conjecture has been given in [7. Note that this parameter can be generalized for higher values of $q$. In [8], Havet et al. prove that, if $G$ is a planar graph, then $\mathcal{T}_{q}(G) \leq q+6$. They also prove that this is best possible if $q \geq 4$, and conjecture that $\mathcal{T}_{3}(G) \leq 8$.

Now, observe that it is not clear whether $G$ always has a spanning tree with a "good" behaviour, i.e., such that $B B C_{q}(G, T)$ is not much larger than $\chi(G)$. Therefore, it makes sense to define the best case behaviour of $B B C_{q}(G, T)$. In [12, Wang, $\mathrm{Bu}$, Montassier and Raspaud asked what is the smallest value $\beta$ for which the following holds: if $G$ is a nonbipartite planar graph with girth at least $\beta$, then $G$ has a spanning tree $T$ such that $B B C_{2}(G, T)=4$. Inspired by their question, we define the following parameter, for a given graph $G$ and a positive integer $q$ :

$\mathcal{B}_{q}(G)=\min \left\{B B C_{q}(G, T): T\right.$ is a spanning tree of $\left.G\right\}$.

Our main result is the following: 
Theorem 2. For every graph $G$ and positive integer $q$,

$$
\mathcal{B}_{q}(G)=\max \left\{\chi(G),\left\lceil\frac{\chi(G)}{2}\right\rceil+q\right\} .
$$

This gives us the following value for bipartite graphs:

Corollary 1. If $G$ is bipartite, then $\mathcal{B}_{q}(G)=q+1$.

Considering $q \geq 2$, observe that if $G$ has at least one edge and $T$ is a spanning tree of $G$, then $B B C_{2}(G, T) \geq 3$, and that $B B C_{2}(G, T)=3$ if, and only if, $G$ is bipartite. Also, observe that, when $G$ is a nonbipartite planar graph, we get that $\max \{\chi(G),\lceil\chi(G) / 2\rceil+2\}$ is always equal to 4 . Therefore, the answer to Wang et al's question is $\beta=3$, i.e., having high girth is not a necessary condition for having the desired spanning tree.

Corollary 2. If $G$ is a nonbipartite planar graph and $q \geq 2$, then $\mathcal{B}_{q}(G)=q+2$. In particular, $G$ always has a spanning tree $T$ for which $B B C_{2}(G, T)=4$.

We mention that this generalizes results in a number of papers: 4, 5, 13, 6, 12. We also mention that, in [12, Wang et al. wrongly state that $\beta$ is at least 4 due to the existence of a nonbipartite planar graph $G$ and a spanning tree $T$ of $G$ such that $B B C_{2}(G, T)=6$. However, they fail to notice that, in order for $\beta$ to be at least 4 , this should hold for every spanning tree of $G$.

\section{Proof of Theorem 2}

Roughly, the idea of the proof is to show that any graph $G$ has a nice proper $k$-coloring, where $k=\max \{\chi(G),\lceil\chi(G) / 2\rceil+q\}$. By nice we mean that the subgraph of $G$ induced by the edges whose endpoint colors differ by at least $q$ form a connected spanning subgraph of $G$. Then, we select among these edges a spanning tree to form its backbone. Before presenting the main result, let us recall some definitions, and present some new ones.

Consider a proper $k$-coloring $c$ of a graph $G$. For $i \in\{1, \ldots, k\}$, the color class $i$ of $c$ is the subset $c_{i}=\{u \in V(G): c(u)=i\}$. Observe that if $H$ is a component of $G\left[c_{i} \cup c_{j}\right]$, a.k.a. Kempe's chain, then the $k$-coloring $c^{\prime}$ obtained from $c$ by switching colors $i$ and $j$ in $V(H)$ is also a proper $k$-coloring of $G$. We denote the set of edges $\{u v \in E(G): u \in V(H)$ and $v \in V(G) \backslash V(H)\}$ by $[H, \bar{H}]$. Given an integer $q$, and $i \in\{1, \ldots, k\}$, we denote by $[i]_{q}$ the set $\{j \in$ $\{1, \ldots, k\}:|i-j|<q\}$. The $q$-subgraph of $c$, denoted by $G_{c, q}$, is the subgraph $\left(V(G), E_{c, q}\right)$, where $E_{c, q}=\{u v \in E(G):|c(u)-c(v)| \geq q\}$. Alternatively, one can see that $u v \in E_{c, q}$ if and only if $c(u) \notin[c(v)]_{q}$ if and only if $c(v) \notin[c(u)]_{q}$. Our upper bound is obtained as a corollary of the following theorem:

Theorem 3. If $G$ is a connected graph and $k \geq \max \{\chi(G),\lceil\chi(G) / 2\rceil+q\}$, then there exists a proper $k$-coloring $c$ of $G$ such that $G_{c, q}$ is connected. 
Proof. Consider $k=\max \{\chi(G),\lceil\chi(G) / 2\rceil+q\}$ and let $c$ be a proper $k$-coloring of $G$ that uses the following $\chi(G)$ colors: $\left\{1, \ldots, x, x+k^{\prime}+1, \ldots, k\right\}$, where $x=\lceil\chi(G) / 2\rceil$ and $k^{\prime}=k-\chi(G)$. Let $H$ be a component of $G_{c, q}$ with maximum number of vertices. Suppose, without loss of generality, that $c$ maximizes the size of $H$. We claim that such a coloring $c$ satisfies that $G_{c, q}$ is connected, which means that $H$ is a spanning subgraph of $G$.

By contradiction, suppose that $V(H) \subset V(G)$, i.e., $H$ does not contain every vertex of $G$. Since $G$ is connected, there must be an edge $u v \in[H, \bar{H}]$. By the definition of $G_{c, q}$, we know that $[c(u)]_{q} \cap[c(v)]_{q} \neq \emptyset$.

First, suppose that there exists $j \in\{1, \ldots, k\} \backslash\left([c(u)]_{q} \cup[c(v)]_{q}\right)$, and let $H^{\prime}$ be the component of $G\left[c_{j} \cup c_{c(v)}\right]$ containing $v$. We claim that $V\left(H^{\prime}\right) \cap V(H)=\emptyset$. Suppose otherwise and let $v^{\prime} \in V\left(H^{\prime}\right) \cap V(H)$ be closest to $v$ in $H^{\prime}$; also, let $w \in N_{H^{\prime}}\left(v^{\prime}\right) \backslash V(H)$ (it exists by the choice of $v^{\prime}$ ). By the definition of $H^{\prime}$, we know that $\left\{c\left(v^{\prime}\right), c(w)\right\}=\{j, c(v)\}$. This contradicts the construction of $H$ since $w v^{\prime} \notin E_{c, q}$ and $j \notin[c(v)]_{q}$. Now, let $c^{\prime}$ be obtained from $c$ by switching colors $j$ and $c(v)$ in $H^{\prime}$. Because $V\left(H^{\prime}\right) \cap V(H)=\emptyset$, nothing changes in $H$; additionally, $c^{\prime}(v) \notin\left[c^{\prime}(u)\right]_{q}$, which means that $u v \in E_{c^{\prime}, q}$ and that there is a component in $G_{c^{\prime}, q}$ that strictly contains $H$, a contradiction to the choice of $c$.

Now, suppose that

$$
[c(u)]_{q} \cup[c(v)]_{q}=\{1, \ldots, k\} \text {, for all } u v \in[H, \bar{H}] .
$$

Recall that $c$ uses the colors that are in the set $\left\{1, \ldots, x, x+k^{\prime}+1, \ldots, k\right\}$, where $k=\max \{\chi(G),\lceil\chi(G) / 2\rceil+q\}, x=\lceil\chi(G) / 2\rceil$ and $k^{\prime}=k-\chi(G)$. We want to prove that $1 \notin[i]_{q}$, for every $i \in\left\{x+k^{\prime}+1, \ldots, k\right\}$, and that $k \notin[i]_{q}$, for every $i \in\{1, \ldots, x\}$. We analyse the cases below.

- $q \geq\lfloor\chi(G) / 2\rfloor$ : in this case, $k=x+q$. If $i \in\{1, \ldots, x\}$, then $k-i \geq$ $k-x=x+q-x=q$. In case, $i \in\left\{x+k^{\prime}+1, \ldots, k\right\}$, then $i-1 \geq$ $x+k^{\prime}+1-1=x+k-\chi(G)=x+x+q-\chi(G) \geq q$.

- $q<\lfloor\chi(G) / 2\rfloor$ : observe that $k=\chi(G)$ and $k^{\prime}=0$. If $i \in\{1, \ldots, x\}$, then $k-i \geq k-x=\chi(G)-x=\lfloor\chi(G) / 2\rfloor>q$. Similarly, if $i \in\left\{x+k^{\prime}+1, \ldots, k\right\}$, then $i-1 \geq x+k^{\prime}+1-1=x>q$.

Now, consider any edge $u v \in[H, \bar{H}]$. Suppose that $c(u) \leq x$, in which case $k \notin$ $[c(u)]_{q}$; if this is not the case, we get $1 \notin[c(u)]_{q}$ and the argument is analogous. By Equation 1, we get $k \in[c(v)]_{q}$, and therefore $c(v) \geq x+k^{\prime}+1$. Let $H^{\prime}$ be the component of $G\left[c_{k} \cup c_{c(v)}\right]$ containing $v$. We claim that $V\left(H^{\prime}\right) \cap V(H)=\emptyset$. Suppose otherwise, and let $v^{\prime} \in V\left(H^{\prime}\right) \cap V(H)$ be the closest to $v$ in $H^{\prime}$ and let $w \in N_{H^{\prime}}\left(v^{\prime}\right) \backslash V(H)$. By the choice of $H^{\prime}$, we know that $\left\{c\left(v^{\prime}\right), c(w)\right\}=\{c(v), k\}$, in which case $1 \notin\left[c\left(v^{\prime}\right)\right]_{q} \cup[c(w)]_{q}$, contradicting Equation [1 Finally, the theorem follows by the same argument used on the previous case.

It remains to prove that this is also a lower bound. Our proof actually holds for any spanning backbone that does not contain isolated vertices. 
Lemma 1. If $G$ is a graph and $H$ is a spanning subgraph of $G$ such that $\delta(H) \geq$ 1, then, for every positive integer $q$ the following holds:

$$
B B C_{q}(G, H) \geq \max \left\{\chi(G),\left\lceil\frac{\chi(G)}{2}\right\rceil+q\right\} .
$$

Proof. Let $H$ be any spanning subgraph of $G$ with $\delta(H) \geq 1$, and let $k=$ $B B C_{q}(G, H)$. Furthermore, let $c$ be a $q$-backbone $k$-coloring of $G$. Since any $q$-backbone coloring of $(G, H)$ is also a proper coloring of $G$, we have that $k \geq \chi(G)$. Now, if either $q \leq\lfloor\chi(G) / 2\rfloor$, or $q \geq\lceil\chi(G) / 2\rceil$ and $k \geq 2 q$, we are done. So, suppose $q \geq\lceil\chi(G) / 2\rceil$ and $k<2 q$, and let $k^{\prime}=2 q-k$. We claim that $[i]_{q}=\{1, \ldots, k\}$, for every $i \in\left\{q-k^{\prime}+1, \ldots, q\right\}$. Because $d_{H}(u) \geq 1$, we know that none of these $k^{\prime}$ colors can be used on $u$, for every $u \in V(G)$, and the following holds:

$$
k-k^{\prime}=k-2 q+k \geq \chi(G) .
$$

This inequality implies that:

$$
k \geq\left\lceil\frac{\chi(G)}{2}\right\rceil+q .
$$

It remains to prove our claim. So, let $i$ be any color in $\left\{q-k^{\prime}+1, \ldots, q\right\}$. It suffices to show that $\{1, k\} \subseteq[i]_{q}$. Clearly, $1 \in[i]_{q}$, since $i \leq q$. Also, since $k=2 q-k^{\prime}$ and $i \geq q-k^{\prime}+1$, we get $k-i \leq 2 q-k^{\prime}-q+k^{\prime}-1=q-1$. Thus, $k \in[i]_{q}$ and the lemma follows.

\section{References}

[1] A. Bondy, U. Murty, Graph Theory, Graduate Texts in Mathematics, Springer-Verlag London, 2008.

[2] H. Broersma, F. V. Fomin, P. A. Golovach, G. J. Woeginger, Backbone colorings for networks, in: H. L. Bodlaender (Ed.), Graph-Theoretic Concepts in Computer Science, Vol. 2880 of Lecture Notes in Computer Science, Springer Berlin Heidelberg, 2003, pp. 131-142.

[3] H. Broersma, F. V. Fomin, P. A. Golovach, G. J. Woeginger, Backbone colorings for graphs: Tree and path backbones, Journal of Graph Theory 55 (2) (2007) 137-152.

[4] Y. Bu, X. Bao, Backbone coloring of planar graphs for $c_{8}$-free or $c_{9}$-free, Theoretical Computer Science 580 (2015) 50-58.

[5] Y. Bu, Y. Li, Backbone coloring of planar graphs without special circles, Theoretical Computer Science 412 (46) (2011) 6464-6468.

[6] Y. Bu, S. Zhang, Backbone coloring for $c_{4}$-free planar graphs, Sci. Sin. Math 41 (2011) 197-206. 
[7] V. Campos, F. Havet, R. Sampaio, and A. Silva. Backbone colouring: Tree backbones with small diameter in planar graphs. Theoretical Computer Science 487 (2013), 50-64.

[8] F. Havet, A.D. King, M. Liedloff, I. and Todinca. (Circular) backbone colouring: Forest backbones in planar graphs. Discrete Applied Mathematics 169 (2014), 119-134.

[9] T. R. Jensen, B. Toft, Graph Coloring Problems, Wiley-Interscience, New York, 1995.

[10] R. Karp, Reducibility among combinatorial problems, in: R. Miller, J. Thatcher (Eds.), Complexity of Computer Computations, Plenum Press, 1972, pp. 85-103.

[11] M. Molloy, B. Reed, Graph Colouring and the Probabilistic Method, 1st Edition, Springer, 2001.

[12] W. Wang, Y. Bu, M. Montassier, A. Raspaud, On backbone coloring of graphs, Journal of combinatorial optimization 23 (1) (2012) 79-93.

[13] S.-M. Zhang, Y.-H. Bu, Backbone coloring for $c_{5}$-free planar graphs, J. Math Study 43 (4) (2010) 315-321. 